\title{
Problem-driven innovation models for emerging technologies
}

\section{Graphical representation of need-led innovation methodologies in healthcare}

\author{
Erfan Soliman $^{1}$ - Daniel Mogefors ${ }^{1}$ • Jeroen H. M. Bergmann ${ }^{1}$ (D)
}

Received: 1 May 2020 / Accepted: 11 June 2020 / Published online: 23 June 2020

(C) The Author(s) 2020

\begin{abstract}
As a fundamentally resource-intensive endeavour, healthcare innovation can benefit from a problem-based approach. This kind of methodology needs to define the problem by applying a range of well-established techniques, such as ethnographic research, market analysis, and stakeholder exploration. However, no in-depth investigation has taken place on how these techniques interact and relate to one another. As such, an overarching methodology is needed in order to represent, critically assess, and evolve problem-driven, or need-led, innovation approaches. Graph theory provides a useful way by which this can be done. This paper exemplifies how different elements of a problem-first approach to innovation can be graphically represented within a system, in order to provide insights into the processes that support real-world impact for new technologies. By providing a more refined description of the need-led innovation methodology, it is hoped that these models can drive a more evidence-based and empirical mindset within the field to ultimately drive valuable innovations with increased efficiency.
\end{abstract}

Keywords Innovation · Graph theory · Emerging technologies · System performance · Medical devices

\section{Introduction}

Bringing technological innovations into the market within healthcare can be resource-intensive, due to regulatory requirements and the relatively high upfront costs of research and development $[1,2]$. This is further complicated by the complexity of rule interpertation that emerges during the regulatory process [3]. Nonetheless, there is high demand for continued innovation, and the spread of new technologies remains relatively unrestrained in many countries [4]. Advanced emerging technologies, such as three-dimensional bioprinting, which can be used to print large tissue structures, auto-injection devices, which can eliminate the use of needles, or new kind of prosthetics are already reaching a stage of preclinical and clinical research $[5,6,19]$. These new technologies can impact a

Jeroen H. M. Bergmann

jeroen.bergmann@eng.ox.ac.uk

1 Natural Interaction Lab, Oxford Institute of Biomedical Engineering, Department of Engineering Science, University of Oxford, Old Road Campus Research Building, OX3 7DQ, Oxford, UK range of healthcare problems, and it will be their 'market pull', as well as the regulations around them, that are likely to be the key factors defining their potential success going forward [7, 8]. Understanding which problems or needs these technologies address is therefore important for the research community, especially from a perspective of resource efficiency.

Methodologies that can reduce the risk connected to healthcare innovation provide value for those interested in creating impact that is economically sustainable. In its simplest form, the progression of innovation can be regarded as a linear process [9]. Even nuanced approaches that compare and propose an integration of the induced, path-dependent, and evolutionary models for innovation suggest that one can define 'sources', or simply starting points, for an innovation cycle [10-12]. We propose that one such point can logically be set to originate at the level of the need, problem, or even question that must be solved or answered. With this logic, defining the need before any solution is introduced would be an appropriate approach to innovation. However, the interest in solutions often outstrips the appeal of critically assessing the problem. This mismatch is often clear in terms of the selection procedures available for each, as it is common practice to 
compare technical solutions, but a critical comparison of any needs is less frequently applied $[10,13]$.

Many examples exist that describe a lack of need assessment, particularly in healthcare, outlining the fact that misinterpreting the problem can have major consequences [1, $14,15]$. One such example was described by Brown when he highlighted how high income countries tended to promote the building of large hospitals in low income countries without addressing the issue of staffing, maintenance, and funding [16]. Brown stated the need to 'particularise the problems and approach the specific manifestations of disease with knowledge of local conditions and resources', which indicates that the system itself can consist of several crucial system elements. This work, which was published in 1966, already showed the importance of understanding the problems that need to be addressed before any solutions are implemented. On the other end of the scale, a problem-based focus has also been shown to create innovative solutions in drug discovery $[17,18]$. These examples highlight the importance of including the problem as a key part of the innovation process. The need-led methodology described herein consists of a systematic method for identifying and describing specific areas of need in a specific population, discovering factors contributing to the perpetuation of problems, and determining criteria to successfully assess potential solutions in an outcome-orientated manner [20]. The methodology aims to provide a clear problem description, or need statement, through critical assessment of all available data, upon which the subsequent solution can be mapped.

The overall activity of applying a need-initiated innovation process in healthcare could be modelled as a system, in order to objectively define the constituent elements of the method. Systems are seen as man-made, created and utilised to provide products or services in defined environments for the benefit of users and other stakeholders [21]. In this case, the system represents the 'service' of building appropriate solutions based on relevant needs. The system itself can consist of several system elements (or attributes) that can be observed and characterised. To formalise this more, we can apply ideas from graph theory, which studies the interacting elements of a system [22].

Thus, the aim of this paper is to propose a graph theory approach for mapping the interactions between established research and innovation domains with regards to need-led innovation. The model is adaptable, and it can be used to study the process and critically assess the proposed interactions. This concept implies that the value of need-led methodologies could lie within the interaction of different domains, providing a modular view that can be assessed more readily. The model can offer insights into how well innovative technologies fit a certain problem.

\section{Theoretical framework}

A potential drawback of approaches to innovation that follow a solution-centric or technology-push path is that they may not capture all of the contextual dependencies and/or subtleties in relationships between various stakeholders, end-users, payers, and beneficiaries of the innovation, particularly when they are affected by factors that may be continually changing [7, 8]. For example, a 2011 paper by Atuahene-Gima and Wei found that the speed and creativity of problem-solving within new product innovations mediated the relationship between market knowledge and competitive advantage [23]. Further, they found that problem-solving speed had a different impact depending on the perceived environment, e.g. if turbulence was perceived as being high, the positive relationship between problemsolving speed and new product performance was stronger. It is difficult for traditional innovation models to objectively capture and support analysis of such dynamic relationships, as it is impacted by factors not represented within the considered system.

Looking back to the work of Usher, which drew insights from Gestalt psychology to frame the cumulative synthesis approach to innovation, wherein radical advancements are seen as coming forth from the aggregation of relatively incremental advancements, the following four steps can be outlined for an individual invention [24]:

1. Perception of the problem: an incomplete or unsatisfactory pattern or method of satisfying a want is perceived;

2. Setting the stage: the elements or data necessary for a solution are brought together through some particular configuration of events or thoughts;

3. Act of insight: the essential solution of the problem is found; and

4. Critical revision: the newly perceived relations become fully understood and effectively worked into the entire context to which they belong, potentially calling for new acts of insight as revisions and improvements are made.

This construction focusses on solutions ('acts of insight') that result from the direct explorations taking place due to problems being initially perceived. While this framework is extended as a general theory to discuss cumulative technological evolution, the model is informative in that it frames the creation of a specific solution to a specific problem domain [18, 24, 25]. This mapping of a unique solution space to a uniquely defined need space, which is a cornerstone of need-led innovation theory, highlights not only the attention that is due to the initial problem at hand, but also the basis that it provides for future explorations 
and improvements to be made. This suports the idea that the evolution of radical innovations is driven by recognising problems [26].

The push towards the establishment of an integrative approach to innovation, inclusive of need-led 'induced' innovation, is not new. In fact, in 1997, Ruttan proposed that each of the three innovation models (induced, evolutionary, and path-dependent) were approaching a dead end, and that a more general theory capable of integrating the three was needed to appropriately model the sources of technical change [10]. While there is of course a lot of discussion, discord, and research about which form of innovation might be best [10-12, 27-29], along with some evidence that the best approach might depend on the specific innovation lifecycle [13], medical innovation, in particular, has been described as benefiting significantly from a 'demand-pull' approach as compared to a 'technology-push' one [30, 31]. This can be attributed to the fact that innovation within healthcare is highly constrained by the trade-off between impact and the resource cost of technological implementation [17]. As such, it is critical that the most impactful technologies are prioritised, for which a deep understanding of the need being targeted is essential. Indeed, Gelijns and Rosenberg describe innovation as fluid, influenced by (1) close interactions between developers and users, (2) changes in financing and delivery modes, and (3) shifting patterns of medical specialisation, which they argue impact the effect of innovation on costs. Further, as stated by Ruiz et al, given that consumers are more often than not faced with problems while using any product or service, a good approach to innovation, whether incremental or otherwise, should involve an identification of strategies to gain as many unique insights as possible from end-users about their needs, behaviours, and responses to stimuli [32]. This should be done long before any innovation path is embarked upon.

As shown, the need-led method can be considered to be multifactorial in nature, and it is therefore important to develop an understanding of the interactions and relationships between different elements. The aim of this paper is to introduce graph-based approaches to explore these nuanced interactions.

\section{Graphical representation of need finding}

\subsection{Methodology}

The interacting elements of a system can be represented graphically, and such a visual representation can support our understanding of a given system. Applying this to the need-led innovation process means that system elements can reflect the aspects of the methodology that are important to achieve desired outcomes. For example, one element can represent the ethnographic research component, or 'immersion', that occurs at the start of the need finding process. Ethnographic research consist of detailed observations in real-world environments. This stage of the process is essential for finding a set of different needs that can be compared. Ethnographic research aims to study social interactions, behaviours, and perceptions that occur within groups, teams, organisations, and communities [33]. Ethnographic research also allows the need-led innovator to remain open-minded about the ideas and problems that they are exploring. This element of the system provides a well-researched method for obtaining observations that can inform needs.

The granularity of each element itself can differ depending on the data available or questions posed. For example, the ethnographic research element can be further split into e.g. informal interviews, images (such as photographs), or field notes. Essentially, the need-led methodology can be described as a system of systems (SoS), and the researcher or innovator can decide at which level the interpretation should take place [22].

From an abstract point of view, the elements can be considered to be nodes or vertices that interact with one another, visually represented by connecting lines or edges. Although this kind of graph is not the only way to represent the system, it can be a very useful approach, especially if we consider the connectivity of the components when describing the SoS [34]. The graph can subsequently provide information about specific aspects of the overall system and, for example, provide insights into the existence of any risks embedded therein. Further analysis of the graph might also offer indications of how to mitigate such risks.

A graph is defined as being formed by vertices and the edges that connect these vertices. At the need finding stage, the methodology can consist of formal interviews, surveys, literature reviews, and ethnographic research (as already discussed). As well as these common forms of research, nodes may also be used to represent some less well-developed methods such as subproblem decomposition [32]. Rather than using traditional questioning to understand a user's problem, subproblem decomposition asks users to think out loud while performing tasks using a given product or service, which generates alternative insights into problems. The number of different elements that are captured in a specific stage is flexible and can be determined by the researchers. It is assumed that there are only finite sets of vertices and edges, a reasonable assumption as there are theoretical limits in terms of the number of elements that can connect to the overall methodology. In addition, we will rely on basic graph descriptors by saying that a graph 
is trivial if there is only one vertex and simple if there are no loops, with each pair of connected vertices only linked by one edge [35].

\subsection{Results and discussion based on simulated data}

An example of a graph that captures the first part of the need-led innovation process is given in Fig. 1. In this graph, the vertices show the original observation $\left(v_{1}\right)$, a survey $\left(v_{2}\right)$, an interview $\left(v_{3}\right)$, a literature review $\left(v_{4}\right)$, and a basic need description $\left(v_{5}\right)$. This provides a simple representation of the 'need finding' stage of the need-led innovation methodology. Vertices $v_{2}$ to $v_{4}$ are part of the information gathering stage that are used to define the need, based on the original observation.

The representation can be further described by stating that the graph can exist as a pair of sets $(V, E)$, where $V$ is the set of vertices and $E$ is the set of edges. The vertices in this case represent the aforementioned elements relevant for constructing a suitable need.

The scenario shown in Fig. 1 consists of sets, where vertices are denoted as $v$ and edges as $e$. The graph can be described with the following equation for $V$, and subsequently for $E$ :

$V=v_{1}, v_{2}, \ldots v_{5}$

$E=\left\{\left(v_{1}, v_{3}\right),\left(v_{1}, v_{2}\right),\left(v_{1}, v_{4}\right),\left(v_{2}, v_{3}\right), \ldots\left(v_{4}, v_{5}\right)\right\}=\left\{e_{1}, \ldots e_{9}\right\}$

Two vertices are adjacent if they are connected by the same edge, e.g. $v_{1}$ and $v_{2}$ (Fig. 1). Working from the edge it can be stated that $e_{2}$ is incident to $v_{1}$ and $v_{2}$. We can extend this to an incidence function $\psi$, which associates each edge with an unordered pair of vertices [35]. The degree of a vertex is given by the number of edges incident to the vertex.
Table 1 Incidence matrix of the graph in Fig. 1. Columns are edges $(e)$ and rows are vertices $(v)$

\begin{tabular}{llllllllll}
\hline & $e_{1}$ & $e_{2}$ & $e_{3}$ & $e_{4}$ & $e_{5}$ & $e_{6}$ & $e_{7}$ & $e_{8}$ & $e_{9}$ \\
\hline$v_{1}$ & 1 & 1 & 1 & 0 & 0 & 0 & 0 & 0 & 0 \\
$v_{2}$ & 0 & 1 & 0 & 1 & 0 & 1 & 1 & 0 & 0 \\
$v_{3}$ & 1 & 0 & 0 & 1 & 1 & 0 & 0 & 1 & 0 \\
$v_{4}$ & 0 & 0 & 1 & 0 & 1 & 1 & 0 & 0 & 1 \\
$v_{5}$ & 0 & 0 & 0 & 0 & 0 & 0 & 1 & 1 & 1 \\
\hline
\end{tabular}

A value of 1 shows that a vertex and edge are incident, whilst a value of 0 indicates that this is not the case

For example, the degree of $v_{2}$ in Fig. 1 is 4 , which can be written as $\operatorname{deg}\left(v_{2}\right)=4$. An incidence matrix can now be obtained for the graph in Fig. 1. This incidence matrix is a representation of the graph-it becomes very useful when there is directionality in the graph (Table 1).

The graph shown in Fig. 1 is an undirected graph, meaning that all the edges are bidirectional. Arrows will be used in the case of a directed graph, or digraph, to show that the edges point in a certain direction [36]. In our case, part of the graph could also be considered as a digraph, because the observation could be fixed and therefore $v_{1}$ will influence $v_{2}, v_{3}$, or $v_{4}$, but not the other way around. However, if the observation itself is seen as an iterative process that is influenced by the other vertices, then it can become an undirected graph again. This iterative process might also introduce subsequent loops into the graph.

Thus, a multigraph, rather than the simple graph used so far, will be useful in representing the need-led innovation process. These are graphs that contain loops and/or multiple edges. Loops are edges that are incident only to a single vertex, i.e. connecting a vertex to itself [36]. As an example, building back on the simple graph in Fig. 1 , it is conceivable that a survey $\left(v_{2}\right)$ would undergo an iterative process of development, as it is used in research
Fig. 1 Graph formed by vertices $\left(v_{1}, v_{2}, \ldots v_{5}\right)$ and edges $\left(e_{1}, e_{2}\right.$, $\left.\ldots e_{9}\right)$. The vetrices represent original observations $\left(v_{1}\right)$, surveys $\left(v_{2}\right)$, interviews $\left(v_{3}\right)$, literature reviews $\left(v_{4}\right)$, and basic need descriptions $\left(v_{5}\right)$

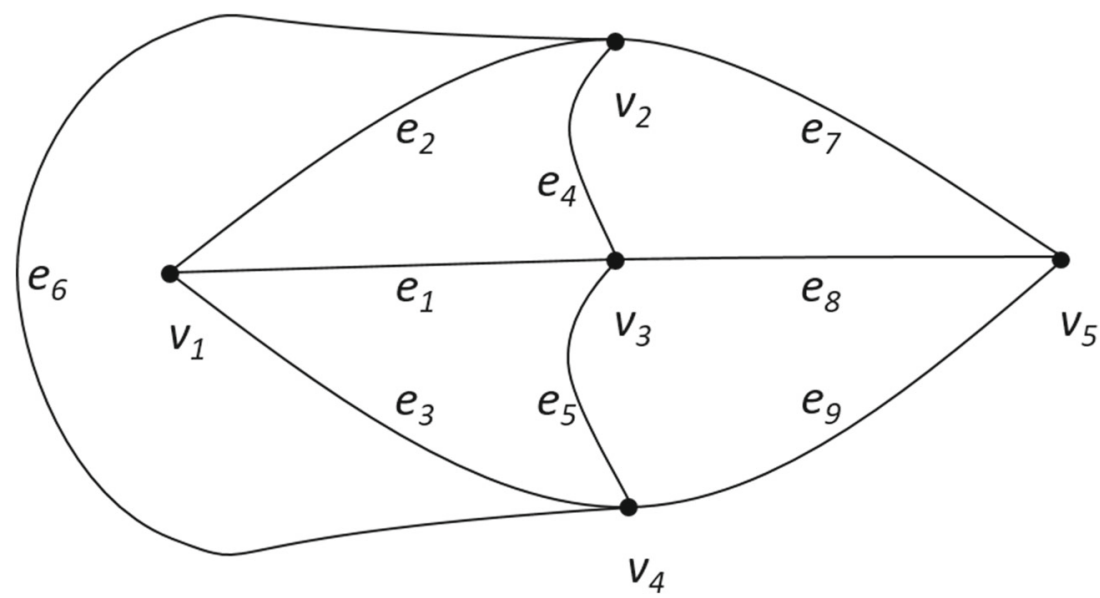


and changes are elicited based on the responses gathered in comparison to the data required.

Multiple, or parallel, edges occur when two vertices are connected via more than one edge. Again, this is helpful in representing the process at hand-a literature review $\left(v_{4}\right)$ may prompt a given approach to an interview $\left(v_{3}\right)$, the data from which may further go on to prompt additional literature reviews to explore certain areas of interest more deeply (here directionality of the edges would be necessary as well).

Thus, in practice, the process of need finding is likely to be more realistically represented by a multigraph that contains 'clusters' of interlinked interviews, surveys, and literature reviews, all of which include iterative loops. For simplicity, at this stage, we can represent an interview, survey, or literature review cluster as a single node, allowing us to maintain the easy-to-visualise representation in Fig. 1.

\section{System-level analysis for need-led innovation graphs}

\subsection{Methodology}

Within graphs, there are often certain subgraphs that are of relevance, such as paths and cycles (it should be noted that these are types of graphs in their own right, and do not necessarily only present as subgraphs) [36]. This notion fits well with the idea of SoS, as graphs can provide inherent extensibility and can model relationships [22], thereby capturing the need-led methodology more accurately than just a simple linear process.

A path is a series of consecutive vertices with a start and end vertex. Each vertex in between connects only with two other vertices, one preceding and one following it. This can most plainly be visualised as a row of vertices connected by single edges. Cycles, put simply, are paths without a start and end vertex - this means that every vertex in a cycle has exactly two neighbours and a degree of 2 (except in the case of a loop, which can be conceived as a cycle containing only a single vertex).

In this paper, we will focus largely on connected graphs, those containing a path via which every vertex in the graph is connected. Within this context, the closeness centrality, or closeness, of a vertex gives information about how connected it is in reference to all other vertices in the graph. This will provide one way by which to identify significant aspects (vertices) of the process that we are modelling.

If the concept of distance between two vertices is defined as the length of the shortest path connecting them, then the farness of a vertex, as defined by the centrality index of a graph [37, 38], is represented by the sum of the distances between the vertex and all others in the graph. Thus, the closeness of a vertex can be calculated as the reciprocal of its farness, such that:

$C\left(v_{i}\right)=\frac{N-1}{\sum_{k-1}^{N} d\left(v_{i}, v_{k}\right)}$

Here $N$ is the total vertex count (minus one in the numerator for the vertex itself) and $d$ is the distance between two vertices $v_{i}$ and $v_{k}$, with $i \neq k$. If there is a need to make comparisons between vertices belonging to graphs of different sizes, then a normalisation step is required. Normalisation is done by taking the average length of the shortest paths represented, multiplied by the total number of vertices in the graph.

Whether a graph is directed or undirected may affect the measure of distance and therefore also the closeness centrality of a vertex, which might have low closeness from incoming connections but high closeness to outgoing ones.

A weighted graph [39] is one in which there is a number (weight) associated with each edge of the graph [40]. Edge-weighted graphs can be helpful for modelling systems whose vertices interact with each other in variable ways, for example when costs, lengths, or capacities are taken into consideration. These graphs can be observed in many contexts, such as in shortest path analyses like the travelling salesman problem [41].

The weight of an edge is factored into measurements of path length and closeness centrality, due to the fact that weights themselves can represent measures of distance, resistance, or efficiency, all of which would have a direct effect on the capacity to traverse a path.

\subsection{Results and discussion based on simulated data}

In Fig. 1, there might be a path $P_{5}$ which has the vertex set $\left\{v_{1}, v_{2}, v_{5}\right\}$ and edge set $\left\{\left(v_{1}, v_{2}\right),\left(v_{2}, v_{5}\right)\right\}$. Highlighting such paths could enable a consideration of the unique influences, relationships, and directionalities that may exist between the constituent vertices, in this case how the orginal observation impacts the survey and in turn how the survey impacts the basic need description [36].

Continuing in the context of our example, $v_{2}, v_{3}$, and $v_{4}$ could be explored as a potential cycle, given that the key interactions that occur between them make up the research phase to identify a basic need. Expanding this to include our previous definition of node clusters, we can begin to obtain the necessary fidelity required for accurately representing the rich and iterative process of need analysis and 'need description' formation. The graph can subsequently be analysed in terms of connectivity to determine the most influential aspects of the process, as well as to potentially identify vulnerabilities within the system. 
In our graph, the closeness centrality is equal to 0.8 for $v_{1}$ and $v_{5}$, whilst it is equal to 1 for all other vertices. The reduced closeness centrality for $v_{1}$ and $v_{5}$ signifies that information dissemination is somewhat more difficult for these two nodes (in this specific graph configuration). Extending the graph will change the information dissemination and subsequently also the closeness centrality values. Going further, edge weights could be used to denote either the cost associated for performing a certain type of analysis on an observation or need statement, or the time required for such an analysis to take place. Both factors of time and cost can be incorporated together to determine a measure of capacity for each edge or node, a concept that we will return to later [42].

\section{Graphical representation of need filtering}

\subsection{Methodology}

When a need has been identified and iterated through several rounds of surveys, interviews, and literature reviews, the next step of the need-led innovation methodology involves an analysis of the commercial viability of the need. This step is conducted after a large number of needs have been generated, so that there is a basis for comparison.

We introduce a 'de-risking' toolkit that can consist of market analysis, stakeholder analysis, and landscape analysis, respectively. We could also include additional vertices for analyses of 'need type/scope' (i.e. does the problem require an incremental change or a blue sky one?), 'disease state fundamentals' (i.e. is the biology of the problem thoroughly understood?), 'feasibility' (i.e. do the technologies required to address the need already exist?), and so on. This concept can be extended in various ways to help de-risk the need as a potential starting point for the building of a solution and ultimately a commercial venture. For the sake of simplicity we will only consider the first three assessment tools.

Iteration of a need through the added graph section outputs a need score, which is a metric by which needs can be 'filtered' down to a list of needs with relatively high commercial value/viability. Need score values might be defined on an ordinal scale of 1 to 3 , indicating the strength of performance for the metric represented in that vertex for a given need.

\subsection{Results and discussion based on simulated data}

We can consider the representation of $v_{5}$ in Fig. 1 and Fig. 2 as a need cluster that contains several well-defined need descriptions, as we introduce a vertex set $\left\{v_{6}, v_{7}\right.$, $\left.v_{8}\right\}$, representing market analysis, stakeholder analysis, and landscape analysis, respectively.

Vertex $v_{5}$ therefore not only represents our initial need cluster, but also our final output cluster, which is made up of iterated, de-risked, and commercially viable needs. By definition, the final output cluster will be significantly smaller than the initial $v_{5}$ cluster that was defined. However, at this stage, we are not concerned about modelling these as different vertices. Our expanded graph can thus be represented by Fig. 2.

Crucially, it should be noted that iteration through the need de-risking toolkit may result in paths that again revisit node clusters $v_{2}, v_{3}$, and $v_{4}$. The process may also include follow-up visits to the initial observation site (e.g. a hospital, in the case of a healthcare observation, or other relevant settings) for additional observations (returning to cluster $\left.v_{1}\right)$. It now becomes important to discuss the directionality of our paths. By iterating through the toolkit vertex set $\left\{v_{6}\right.$, $\left.v_{7}, v_{8}\right\}$, the model implies a certain bidirectionality as far as the edges between these vertices and $v_{5}$ are concerned. In fact, given the application of our current graph, all of the edges drawn thus far can be considered bidirectional. That said, in the case of paths that revisit vertices $\left\{v_{1}, v_{2}, v_{3}, v_{4}\right\}$, this can be interpreted in two ways. In one case, these paths can be considered directional, where a follow-up observation essentially results in a new need statement that must be defined through additional interviews, research, and surveys. On the other hand, a follow-up observation can be used simply as a mechanism for fine-tuning an existing need description, thereby implying bidirectionality. For the
Fig. 2 Expanded graph taking into consideration the representation of need analysis and filtering (in red). Vertices are labelled as $\left\{v_{5}, v_{6}, v_{7}, v_{8}\right\}$ and edges are $\left\{e_{10}, e_{11}, e_{12}\right.$, $\left.e_{13}\right\}$. The vetrices represent basic need descriptions $\left(v_{5}\right)$, market analyses $\left(v_{6}\right)$, stakeholder analyses $\left(v_{7}\right)$, and landscape analyses $\left(v_{8}\right)$

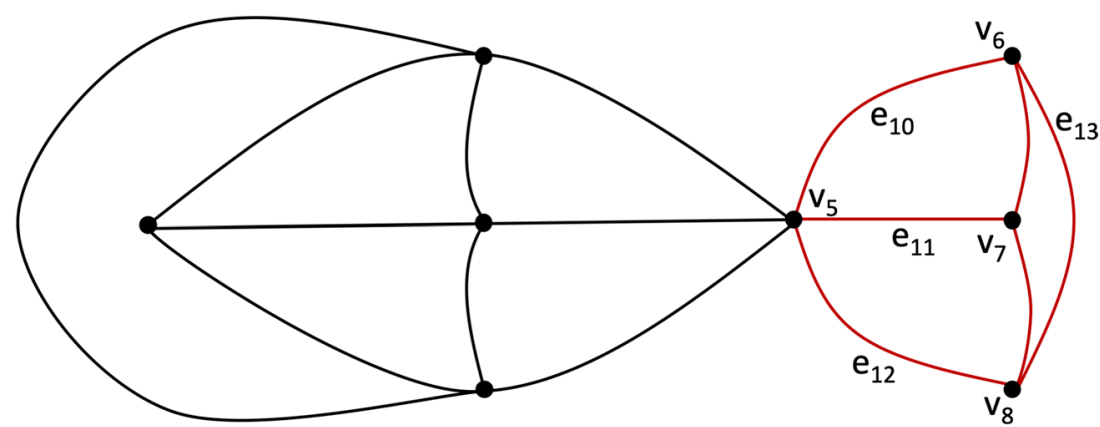


Fig. 3 Further expanded graph. Dashed lines are introduced to denote adjacent possible edges. The adjacent possible edge $\left(e_{14}\right)$ connects the need description cluster $v_{5}$ with the observation cluster $v_{1}$

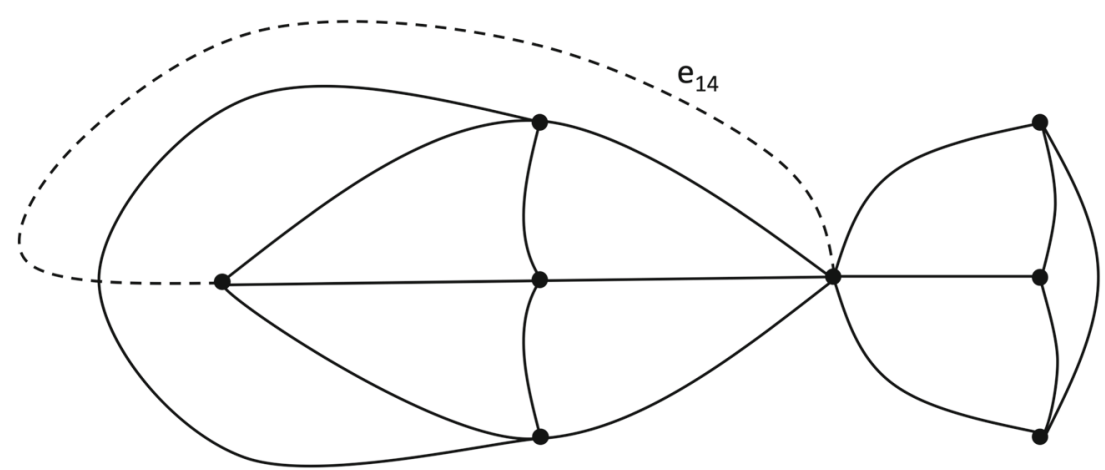

\subsubsection{Path length calculations}

However, a dashed line will be used to denote the fact that this path is only 'active' for bidrectional use once the initial path, e.g. $v_{1}$ to $v_{5}$, has been traversed. Using this description, the edge connecting $v_{5}$ to $v_{1}$ can be considered an 'adjacent possible' edge that only becomes available upon exploration of node $v_{5}$ itself [43-45]. Dashed lines have now been added to the graph for completeness (Fig. 3).

The introduced model provides a clear framework that shows how the need-led innovation methodology is structured. More importantly, it provides the opportunity to further compare, assess, and optimise the process.

In Fig. 4, we expand the graph to include edges connecting each of the need analysis nodes back to the need identification nodes defined previously. This final graph meets the definition of a strongly connected graph-any two vertices are connected by a path in both directions [22]. As an example, we can define both an incoming path to $v_{8}$ from $v_{1}$, and an outgoing path from $v_{8}$ to $v_{1}$ (Fig. 5).
With a working model that maps the process of needs identification and filtering/selection, we can proceed to conduct a system level analysis of the relationships within the model. A simple analysis to begin with involves the measuring of path lengths within the graph, as defined by the number of edges that exist between two vertices. Given that there may be more than one way to connect two vertices, taking into account the directionality of the graph as well as adjacent possible edges, for the purposes of our analysis we will consider both average path lenghts (Table 2) as well as exact path lengths (Table 3). The average path is independent of the direction of each path, while the exact path will create two possibilities depending on which of the vertices is the start of the path, e.g. $v_{1}$ to $v_{8}$ or $v_{8}$ to $v_{1}$. It should be noted that even though this yields different values for 'distance', it is still consistent with our definition, given that each value represents the shortest

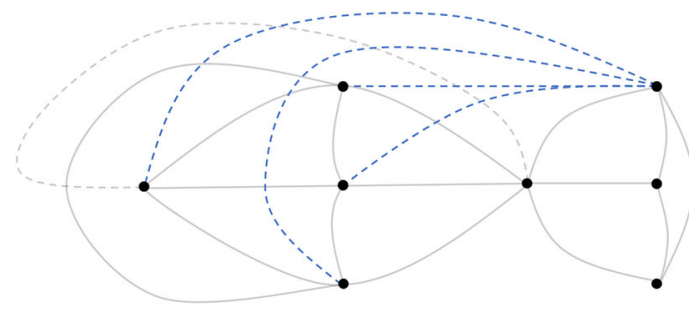

a

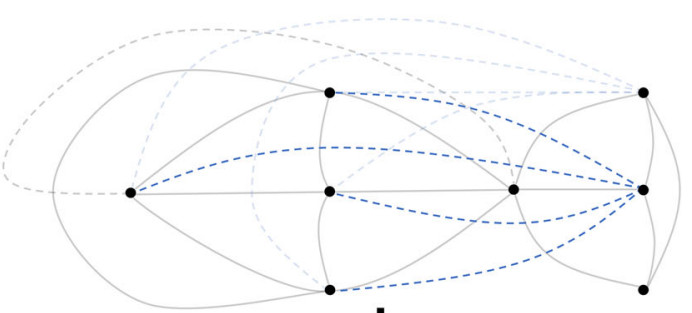

b

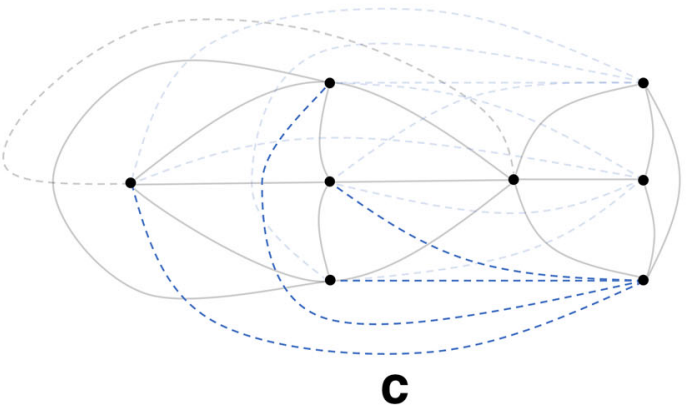

Fig. 4 Further expanded graphs. Dashed lines are introduced to denote adjacent possible edges: (a) adjacent possible edges from market analysis vertex $v_{6}$, (b) adjacent possible edges from stakeholder analysis vertex $v_{7}$, and (c) adjacent possible edges from landscape analysis vertex $v_{8}$ 
Fig. 5 Final graph, based on the previous shown figures, used for path length calculations. Dashed lines denote and adjecent possible edges. $v_{6}$ represents market analysis vertex with adjecent possible edges, $v_{7}$ represents stakeholder analysis vertex with adjecent possible edges, and $v_{8}$ represents landscape analysis vertex with adjecent possible edges

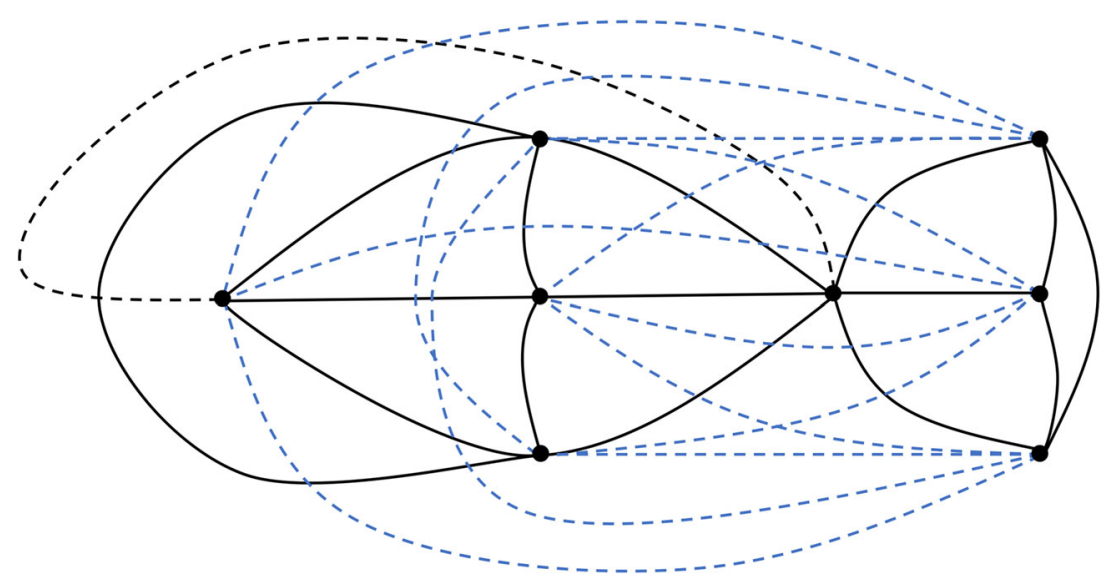

\subsubsection{Network analysis}

possible path connecting two vertices in the direction being considered. Taking the sum of all distances for each vertex yields its 'farness', and dividing that from the number of vertices in the graph allows us to attain a value for the closeness centrality of each vertex, as per (3).

\subsubsection{Centrality of the need}

By simple inspection, we can observe that vertex $v_{5}$, which represents the need vertex, lies at the heart of this sample graph (Table 4). While this depends to some extent on the way each of the vertices are defined and how connections are made, it seems a fitting conclusion for a graph designed to allow for need identification, fine-tuning, and filtering. The importance of the need cluster can be shifted once further stages are added that deal with finding a suitable (technological) solution and (commercial) implementation. The urgency of the need can subsequently drive these next stages, but the suitability of the technology or the ease of its implementation can also influence the need cluster once more. Emerging technologies can thus open up the possibility to pursue new needs that previously were not solvable. This is an interesting interplay that can be well understood through the application of the proposed model.

Table 2 Table of average path lengths in Fig. 5

\begin{tabular}{lllllllll}
\hline & $v_{1}$ & $v_{2}$ & $v_{3}$ & $v_{4}$ & $v_{5}$ & $v_{6}$ & $v_{7}$ & $v_{8}$ \\
\hline$v_{1}$ & - & 1 & 1 & 1 & 1.5 & 2 & 2 & 2 \\
$v_{2}$ & 1 & - & 1 & 1 & 1 & 1.5 & 1.5 & 1.5 \\
$v_{3}$ & 1 & 1 & - & 1 & 1 & 1.5 & 1.5 & 1.5 \\
$v_{4}$ & 1 & 1 & 1 & - & 1 & 1.5 & 1.5 & 1.5 \\
$v_{5}$ & 1.5 & 1 & 1 & 1 & - & 1 & 1 & 1 \\
$v_{6}$ & 2 & 1.5 & 1.5 & 1.5 & 1 & - & 1 & 1 \\
$v_{7}$ & 2 & 1.5 & 1.5 & 1.5 & 1 & 1 & - & 1 \\
$v_{8}$ & 2 & 1.5 & 1.5 & 1.5 & 1 & 1 & 1 & - \\
\hline
\end{tabular}

Given the iterative nature of the need analysis component of the graph, we can opt to visualise this through a network instead. As defined by Harrison in 2016, a network is a multigraph $G$ with source node $s$ and sink node $t$, defined as two explicit vertices of the graph and a mapping $c$ from the edge set to the natural numbers, where the particular value of $c$ for a specific edge is termed its capacity. A network is then the tuple $N:(G, s, t, c)$ [22].

In Fig. 6a, we have defined a source and sink vertex for the set of needs $N$, representing need statements derived from the first component of our graph, which began as simple observations (not depicted here for the purposes of our network representation), 'flowing' through to final need statements that have been fine-tuned and validated through thorough market $(M)$, stakeholder $(S)$, and landscape $(L)$ analyses. A vertex $F$ has been added to represent the filtration that occurs when the results of all analyses are combined and processed to yield a selection of validated and potentially early commercialisable needs. Fig. $6 \mathrm{~b}$ expands the $M$ and $S$ vertex clusters to depict different types of market analyses, top-down $\left(M_{1}\right)$ and bottom-up $\left(M_{2}\right)$, as well as different stakeholder analyses, in depth interviews or

Table 3 Table of exact path lengths in Fig. 5, with incoming and outgoing paths considered separately

\begin{tabular}{lcccccccc}
\hline & $v_{1}$ & $v_{2}$ & $v_{3}$ & $v_{4}$ & $v_{5}$ & $v_{6}$ & $v_{7}$ & $v_{8}$ \\
\hline$v_{1}$ & - & 1 & 1 & 1 & 2 & 3 & 3 & 3 \\
$v_{2}$ & 1 & - & 1 & 1 & 1 & 2 & 2 & 2 \\
$v_{3}$ & 1 & 1 & - & 1 & 1 & 2 & 2 & 2 \\
$v_{4}$ & 1 & 1 & 1 & - & 1 & 2 & 2 & 2 \\
$v_{5}$ & 1 & 1 & 1 & 1 & - & 1 & 1 & 1 \\
$v_{6}$ & 1 & 1 & 1 & 1 & 1 & - & 1 & 1 \\
$v_{7}$ & 1 & 1 & 1 & 1 & 1 & 1 & - & 1 \\
$v_{8}$ & 1 & 1 & 1 & 1 & 1 & 1 & 1 & - \\
\hline
\end{tabular}




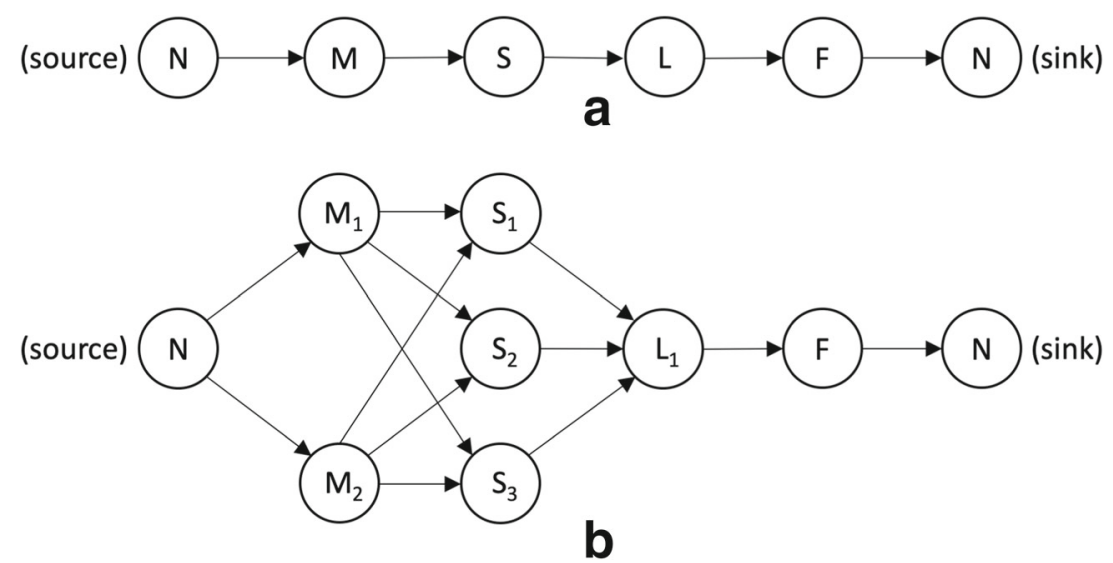

Fig. 6 Need analysis and filtering as a network. (a) The vertex $N$ contains a set of needs that flow through stages of market analyses $M$, stakeholder analyses $S$, and landscape analyses $L$ to reach a filtered state $F$ that yields a set of final needs. (b) The vertex for market analyses can be further seperated, with $M_{1}$ taking a top-down approach

re-immersions $\left(S_{1}\right)$, shorter interviews or phone calls $\left(S_{2}\right)$, and simple online research $\left(S_{3}\right)$. With this representation, we can begin to think about the capacity that exists within the network.

Capacity labels within a network must all be defined using a consistent unit. For example, in our case, we can report capacity as the number of needs per day for which a certain type of analysis can be performed by one person. The need filtering stage could include hunderds of needs that must be considered and processed. For illustration purposes, we can consider that certain number of needs per node can be processed daily. It can be suggested that a basic top-down market analysis can be completed for 7 needs if conducted and $M_{2}$ a bottom-up. Stakeholder analyses can be split into in-depth interviews or re-immersions $S_{1}$, shorter interviews or phone calls $S_{2}$, and online research $S_{3} . L_{1}$ represents a single approach to landscape analyses to be conducted, but others could be added

by a full-time innovator. For bottom-up market analysis, we will use the capacity number of 3 needs. These values are of course just placeholders-real values will depend on the requirements, resources, and the nature of the needs themselves. However, modelling this process as a network is useful for identifying bottlenecks or capacity limitations on a conceptual level.

Using contrived estimates for illustrative purposes, in Fig. 7, we add sample capacity values to the edges connecting our nodes, in order to conduct a max flow analysis. Capacity number estimates are based on how timeintensive a certain type of analysis might be. For example, a thorough stakeholder analysis $\left(S_{1}\right)$ that involves a long
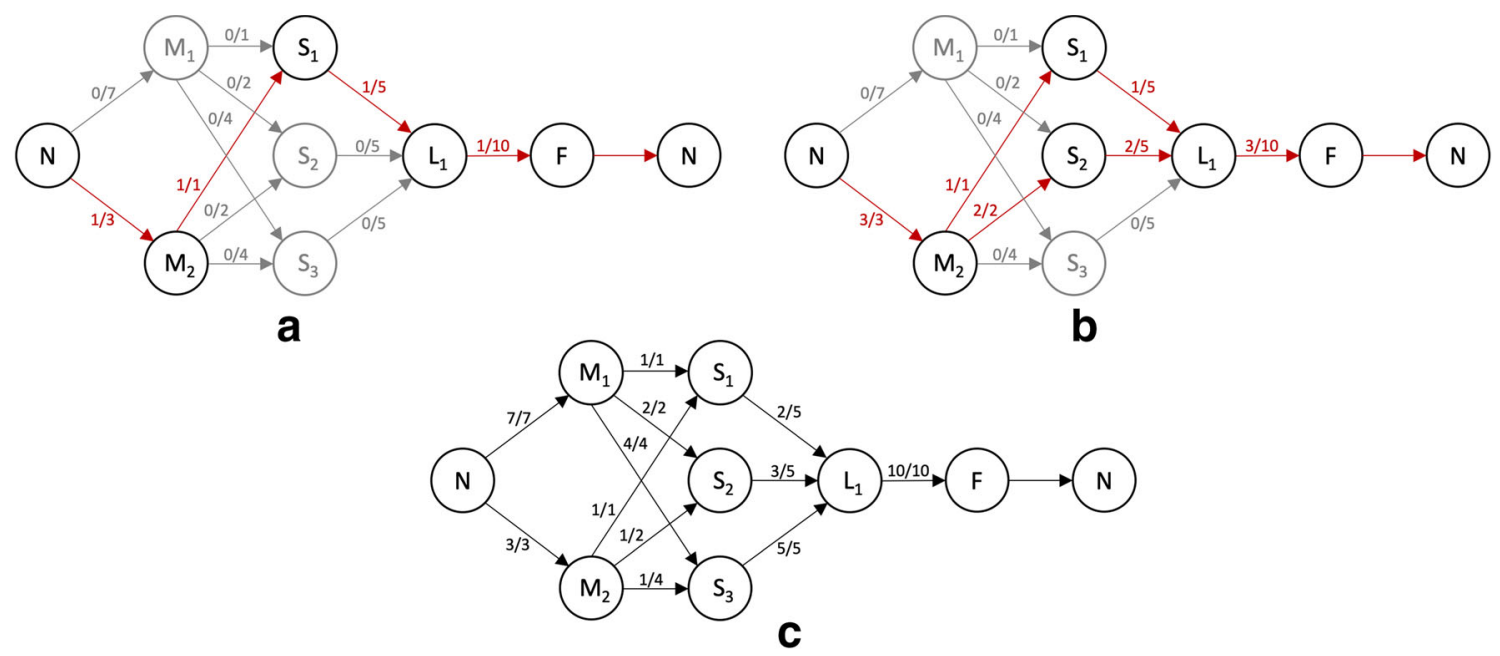

Fig. 7 Analysis and filtering of needs $(N)$ as a network, with market analyses ( $M_{1}$ for top-down and $M_{2}$ for bottom-up), stakeholder analyses (with in-depth interviews or re-immersions represented by $S_{1}$, short interviews or phone calls given by $S_{2}$, and online research as $S_{3}$ ), and landscape analysis $\left(L_{1}\right)$. There is also an added filtering $(F)$ step, used to determine the max flow. The edges show the current capacity and the capacity limit. A gradual build up of capacity is shown in (a) and (b) through the selected flow path coloured red. Maximum flow for the whole network is shown in (c) 
Table 4 The sum of path 'distances', or farness, of each vertex. Values for outgoing, incoming, as well as average farness are given

\begin{tabular}{lllllllll}
\hline & $v_{1}$ & $v_{2}$ & $v_{3}$ & $v_{4}$ & $v_{5}$ & $v_{6}$ & $v_{7}$ & $v_{8}$ \\
\hline Outgoing & 14 & 10 & 10 & 10 & 7 & 7 & 7 & 7 \\
Incoming & 7 & 7 & 7 & 7 & 8 & 12 & 12 & 12 \\
Average & 10.5 & 8.5 & 8.5 & 8.5 & 7.5 & 9.5 & 9.5 & 9.5 \\
Closeness Centrality & 0.67 & 0.82 & 0.82 & 0.82 & 0.93 & 0.74 & 0.74 & 0.74 \\
\hline
\end{tabular}

The closeness centrality of each vertex is also shown based on average path lenghts and a total of eight node clusters face-to-face interview with a stakeholder (or a visit to a hospital setting i.e. re-immersion) may only be possible for one need on a given day, while an analysis that involves online research $\left(S_{3}\right)$ could allow for four needs to be processed. We assume the existence of a specific person at each node (apart from vertex $N$ ) to complete the work assigned for that day (Table 4).

In Fig. 7a, one possible flow path is coloured in red, with the max flow through the path determined by the capacity-limiting edge between $M_{2}$ and $S_{1}$. In Fig. $7 \mathrm{~b}$, a second path is added, depicting one possible configuration for the max flow of needs through $M_{2}$. Fig. 7c uses all paths and redistributes numbers to allow for max flow within the whole network. Please note that in our example, it is possible to achieve max network flow through multiple different configurations, but this may not always be the case [46].

Another way to consider capacity is to look at limitations on the nodes, rather than on the edges, either upon in- or output. In our example of a system that is designed to filter needs by conducting analyses of type $M, S$, and $L$, this is a more realistic representation. This is because the per day limit is specific to the type of analysis, rather than to the channel through which the analysis is done. Said in another way, the $S_{1}$ node cannot receive an input from each of the $M$ nodes as before, but rather one input overall, since the capacity to perform a stakeholder analysis is limited by the time and resource constraints related to that specific activity. With this redefinition, our graph can be represented as in Fig. 8, where an example of max flow has been established based on the maximum capacity of each node. It has to be noted that there are more combinations available for achieving max flow. We can now clearly see that with the capacity numbers previously selected, the flow-limiting section of the graph has shifted to the $L$ node. This shows that our limitation analysis will depend on the graphical representation chosen, and will vary on a case-by-case basis given the capacity bounds that exist.

These models can subsequently help with the planning and scheduling of the process, in addition to its optimisation.

\section{Conclusion}

Topology, graphs, and their study have received a lot of attention due to their ability to represent the realworld in a manner that can be analysed objectively [34]. In this paper, the model presented aims to promote an evidence-based approach to need-led innovation. It offers an opportunity to critically assess the connection between individual elements, as well as the number of elements that make up the methodology. As demonstrated in this paper, any over-reliance on specific vertices can be identified

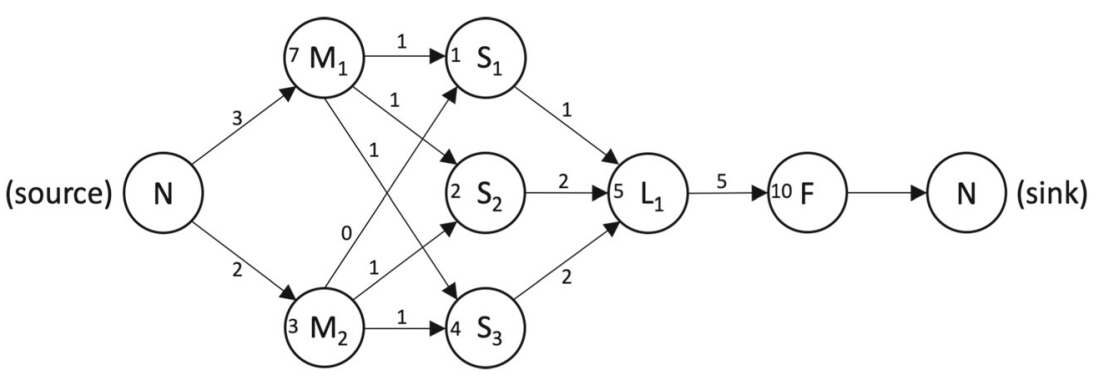

Fig. 8 Need analysis and filtering as a network, modelled with capacities on nodes rather than edges. Numbers listed on edges represent the quantity of needs passing through, and the number listed on the left side of each node represents a full-time innovator's capacity for conducting that type of analysis on a given day. Vertices representing needs $(N)$, market analyses ( $M_{1}$ for top-down and $M_{2}$ for bottom-up), stakeholder analyses (with in-depth interviews or re-immersions represented by $S_{1}$, short interviews or phone calls given by $S_{2}$, and online research as $S_{3}$ ), landscape analysis $\left(L_{1}\right)$, as well as filtering $(F)$ are included in this network 
and a risk-benefit discussion regarding this reliance can be proposed. More importantly, the elements identified can be further scrutinised in terms of evidence supporting their usefulness within the innovation process. The model also provides a framework through which different networks for suggested innovation processes can be described and thus compared. This framework can be further extended using a range of suitable algorthmic techniques [47].

The introduced model can be extended to include a graphical representation of concept generation and selection. This would follow the same logic as described in this paper for the need finding and filtering stages. The suggested need-led methodology model will support the development of an evidence-based innovation framework that can clearly be described to relevant stakeholders. More importantly, it will provide a much needed tool for the critical assessment of approaches within the (need-led) innovation community. The ability to describe, compare, and unify the most suitable problem-based processes will be of great benefit for those who want to optimise the impact and sustainability of their technology [48, 49].

There are a number of limitations of this methodology. Due to its focus on the identification and analysis of needs, the obvious metric for success of the methodology would be confined to the quality and importance (in terms of potential real-world impact) of the needs. For any use case of the graphical representation, then, it may be helpful for researchers to identify longer-term relationships, e.g. to what extent does market analysis predict the market value of the solution that is developed, as a result of the upfront need analysis that is undertaken. This could allow people to develop a better understanding of the long-term value of the various elements they may represent within their system. It is to be seen whether this methodology has utility for such considerations.

Another difficulty that the methodology outlined in the paper presents is its dynamic nature. While this is also one of its benefits, its ability to continually change and be updated with additional nodes and relationships could make the overall model cumbersome and difficult to interpret. In cases of such opacity, objective values such as closeness centrality could aid interpretation and discussion of the model. Even so, the ever-changing nature of the representation could make it difficult to track the value of and interactions between nodes across time, given that chronology is not captured in the graphs. For example, an element such as ethnographic research may be particularly valuable for need identification initially. Yet, the value of ethnographic research may diminish once additional research, e.g. end-user interviews, are added to contribute to need development, with no record that it had initially contributed significantly to identifying the needs that were subsequently further fine-tuned through end-user interviews. Versioning of graphs could perhaps be a way through which to overcome this challenge.

To conclude, graph-thinking, as applied to needled innovation, could offer a new way to study and guide discoveries. This paper suggests a comprehensive framework to develop the next generation of innovative processes informing which problems should be tackled and how new technologies, such as bioprinting [50], medical additive manufacturing [51], or cell-scaffold designs [52, 53] can be optimised to address important needs. This work aims to promote critical development of the field of needled innovation and to create a strategy that can be applied to global challenges and emerging technologies. That said, further considerations and studies regarding the value of this framework in various contexts are needed, and they will be crucial to its development.

Acknowledgements We would like to acknowledge the support and funding from the European Institute of Innovation \& Technology (EIT) Health and the Engineering and Physical Sciences Research Council (EPSRC) in conducting this work. The research was also supported by the National Institute for Health Research (NIHR) Oxford Biomedical Research Centre (BRC).

\section{Compliance with Ethical Standards}

Conflict of interests The authors declare that they have no conflicts of interest.

Consent for Publication No research involving human participants and/or animals was conducted for the purposes of this work.

Open Access This article is licensed under a Creative Commons Attribution 4.0 International License, which permits use, sharing, adaptation, distribution and reproduction in any medium or format, as long as you give appropriate credit to the original author(s) and the source, provide a link to the Creative Commons licence, and indicate if changes were made. The images or other third party material in this article are included in the article's Creative Commons licence, unless indicated otherwise in a credit line to the material. If material is not included in the article's Creative Commons licence and your intended use is not permitted by statutory regulation or exceeds the permitted use, you will need to obtain permission directly from the copyright holder. To view a copy of this licence, visit http:// creativecommonshorg/licenses/by/4.0/.

\section{References}

1. Rettig RA. Medical innovation duels cost containment. Health Aff. 1994;13(3):7-27.

2. Kleinke J, McGee N. Breaking the bank: Three financing models for addressing the drug innovation cost crisis. Ame Health Drug Benef. 2015;8(3):118.

3. Bergmann JH, Hendricusdottir R, Lee R. Regulatory navigation: a digital tool to understand medical device classification pathways. Comprehen Biotechnol. 2019;5:167-172.

4. Bodenheimer T. High and rising health care costs. part 2: Technologic innovation. Ann Intern Med. 2005;142(11):932-937. 
5. Ahadian S, Khademhosseini A. A perspective on 3D bioprinting in tissue regeneration. Bio-Design Manufact. 2018;1(3):157-160.

6. Soliman E, Ranjan S, Xu T, Gee C, Harker A, Barrera A, Geddes J. A narrative review of the success of intramuscular gluteal injections and its impact in psychiatry. Bio-Design Manufact. 2018;1(3):161-170.

7. Roback K, Gäddlin P-O, Nelson N, Persson J. Adoption of medical devices: Perspectives of professionals in swedish neonatal intensive care. Technol Health Care. 2007;15(3):157-179.

8. Kimberly JR, Evanisko MJ. Organizational innovation: the influence of individual, organizational, and contextual factors on hospital adoption of technological and administrative innovations. Acad Manag J. 1981;24(4):689-713.

9. Godin B. The linear model of innovation: the historical construction of an analytical framework. Sci Technol Human Values. 2006;31(6):639-667.

10. Ruttan VW. Induced innovation, evolutionary theory and path dependence: Sources of technical change. Econ J. 1997;107(444):1520-1529.

11. Ruttan VW. Induced innovation and path dependence: a reassessment with respect to agricultural development and the environment. Technol Forecast Soc Chang. 1996;53(1):41-59.

12. Von Tunzelmann N, Malerba F, Nightingale P, Metcalfe S. Technological paradigms: Past, present and future. Ind Corp Chang. 2008;17(3):467-484.

13. Walsh V. Invention and innovation in the chemical industry: Demand-pull or discovery-push?. Res Policy. 1984;13(4):211-234.

14. Baker L, Birnbaum H, Geppert J, Mishol D, Moyneur E. The relationship between technology availability and health care spending: Attempts to address technology availability and rising costs could end up badly misguided if implications for quality are not considered. Health Affairs. 2003;122(Supp11):W3-537.

15. Bodenheimer T. High and rising health care costs. part 1: Seeking an explanation. Ann Intern Med. 2005;142(10):847-854

16. Brown RE. Medical problems of the developing countries. Science. 1966;153(3733):271-275.

17. Gelijns A, Rosenberg N. The dynamics of technological change in medicine. Health Aff. 1994;13(3):28-46.

18. Coccia M. Sources of technological innovation: Radical and incremental innovation problem-driven to support competitive advantage of firms. Technol Anal Strat Manage. 2017;29(9):10481061.

19. Diment LE, Thompson MS, Jeroen HMB. "Three-dimensional printed upper-limb prostheses lack randomised controlled trials: A systematic review". Prosthetics and orthotics international. 2018;42(1):7-13

20. Levy S, Bradley DA, Swanston MT. The technology prescription: Linking telecare and informatics by using a need-led paradigm. Health Inform J. 2002;8(2):88-94.

21. Walden DD, Roedler GJ, Forsberg K, Hamelin RD, Shortell TM, (eds). 2015. Systems Engineering handbook: A Guide for System Life Cycle Processes and Activities, 4th ed. New York: Wiley.

22. Harrison WK. The role of graph theory in system of systems engineering. IEEE Access. 2016;4:1716-1742.

23. Atuahene-Gima K, Wei Y. The vital role of problem-solving competence in new product success. J Prod Innov Manag. 2011;28(1):81-98.

24. Ruttan VW. Usher and schumpeter on invention, innovation, and technological change. Quart J Econ 596-606. 1959.

25. Usher AP. A history of mechanical inventions: revised edition courier corporation. 2013.

26. Coccia M. Problem-driven innovations in drug discovery: coevolution of the patterns of radical innovation with the evolution of problems. Health Policy Technol. 2016;5(2):143-155.

27. Dixon H. Controversy: The source and measurement of technical change: Editorial note. Econ J. 1997;107(444):1518-1519.
28. Consoli D, Mina A. An evolutionary perspective on health innovation systems. J Evol Econ. 2009;19(2):297.

29. Nakićenović N, Nordhaus WD, et al. Technological change and the environment resources for the future. 2002.

30. Singer DR, Marsh A. Challenges and solutions for personalizing medicines. Health Policy Technol. 2012;1(1):50-57.

31. Rogers EM. Diffusion of innovations simon and schuster. 2010.

32. Ruiz D, Jain D, Grayson K. Subproblem decomposition: an exploratory research method for effective incremental new product development. J Prod Innov Manag. 2012;29(3):385-404.

33. Reeves S, Kuper A, Hodges BD. Qualitative research methodologies: Ethnography. Br Med J. 2008;337:a1020.

34. Marvin JW, Garrett RK Jr. Quantitative SoS architecture modeling. Procedia Comput Sci. 2014;36:41-48.

35. Bondy JA, Murty USR, et al. Graph Theory with Applications. 1976, Vol. 290: Macmillan, London.

36. Bondy JA. Basic graph theory: Paths and circuits. Handbook of Combinatorics. 1995;1:3-110.

37. Sabidussi G. The centrality index of a graph. Psychometrika. 1966;31(4):581-603.

38. Carstens L, leidner JL, Szymanski K, Howald B. Modeling company risk and importance in supply graphs. In: European Semantic Web Conference. New York: Springer; 2017. p. 18-32.

39. Strang G. Linear Algebra and its Applications Brooks/Cole Publishing Co. 2005.

40. Fletcher P, Hoyle HB, Patty CW. Foundations of Discrete Mathematics. Pacific Grove: Brooks/Cole Publishing Co.; 1990.

41. Rosenkrantz DJ, Stearns RE, Lewis PM II. An analysis of several heuristics for the traveling salesman problem. SIAM J Comput. 1977;6(3):563-581.

42. Deo N. Graph theory with applications to engineering and computer science courier dover publications. 2017.

43. Felin T, Kauffman S, Koppl R, Longo G. Economic opportunity and evolution: beyond landscapes and bounded raionality. Strat Entrepren J. 2014;8(4):269-282.

44. Gregersen NH. From complexity to life: on the emergence of life and meaning. Oxford: Oxford University Press; 2002.

45. Mitleton-Kelly E. Complex systems and evolutionary perspectives on organisations: the application of complexity theory to organisations elsevier science ltd. 2003.

46. Ford LR, Fulkerson DR. Maximal flow through a network, in Classic Papers in Combinatorics. 2009: Springer, New York.

47. Malliaros FD, Giatsidis C, Papadopoulos AN, Vazirgiannis M. The core decomposition of networks: Theory, algorithms and applications. VLDB J. 2020;29(1):61-92.

48. Teece DJ. Dosi's technological paradigms and trajectories: Insights for economics and management. Ind Corp Chang. 2008;17(3):507-512.

49. Dosi G. Technological paradigms and technological trajectories. Res Policy. 1982;2(3):I47-62.

50. Murphy SV, Atala A. 3D Bioprinting of tissues and organs. Nat Biotechnol. 2014;32(8):773.

51. Diment LE, Thompson MS, Bergmann JH. Clinical efficacy and effectiveness of 3D printing: A systematic review. 2017, Vol. 7.

52. Chui C-Y, Odeleye A, Nguyen L, Kasoju N, Soliman E, Ye H. Electrosprayed genipin cross-linked alginate-chitosan microcarriers for ex vivo expansion of mesenchymal stem cells. J Biomed Mater Res Part A. 2019;107(1):122-133.

53. Soliman E, yang SC, Dombi GW, Bhatia SK. Electrically conductive, biocompatible composite containing carbon nanobrushes for applications in neuroregeneration. In: 2012 38th Annua Northeast Bioengineering Conference (NEBEC), pp. 343-344 IEEE; 2012.

Publisher's note Springer Nature remains neutral with regard to jurisdictional claims in published maps and institutional affiliations. 